\title{
Acute Gallbladder Necrosis in a Patient with Acute Type B Aortic Dissection
}

\author{
Toshiro Ito, MD, PhD, Naomi Yasuda, MD, Yohsuke Kuroda, MD, Motoshi Sugawara, MD, \\ Tetsuya Koyanagi, MD, and Tetsuya Higami, MD, PhD
}

\begin{abstract}
Although vascular complications induced by acute aortic dissection are varied and common, gallbladder necrosis induced by acute aortic dissection is rare. We experienced the case of a 42-year-old woman who suffered from acute gallbladder necrosis that occurred the following day after the onset of acute type B aortic dissection. Contrasted computed tomography, which showed the thickened wall of the gallbladder and the pericholecystic fluid, as well as the occluded celiac artery, was an effective diagnostic procedure. We performed cholecystectomy and revascularization of the celiac artery using autologous saphenous vein. Her postoperative course was uneventful, and she was discharged after 20 postoperative days.
\end{abstract}

Keywords: acute gallbladder necrosis, visceral malperfusion, acute aortic dissection

\section{INTRODUCTION}

A ortic branch vessel obstruction associated with acute aortic dissection manifests as ischemia of various terminal organs, such as the brain, gut, kidney, and legs. However, gallbladder necrosis in patients with acute aortic dissection is rare. We present a case of acute gallbladder necrosis in a patient with acute aortic dissection.

\section{Case Report}

A 42-year-old woman with severe back pain and left lower limb pain was admitted to our hospital. On admission, she was hemodynamically stable; however, the right infrainguinal pulse was not palpable, and her right leg appeared severely pale with accompanying sensory loss. Contrasted computed tomography (CT) showed acute Stanford type B aortic dissection extending from the left

Department of Cardiovascular Surgery, Sapporo Medical University, School of Medicine, Sapporo, Hokkaido, Japan

Received: August 12, 2013; Accepted: September 27, 2013 Corresponding author: Toshiro Ito, MD, PhD. Department of Cardiovascular Surgery, Sapporo Medical University, School of Medicine, South-1 West-16, Chuo-ku, Sapporo,

Hokkaido 080-8543, Japan

Tel: +81-11-611-2111, Fax: +81-11-613-7318

E-mail: t.ito@sapmed.ac.jp subclavian artery to the abdominal aortic bifurcation. The celiac artery (CA) was dissected and occluded by the thrombosed false lumen at the orifice (Fig. 1B). The superior mesenteric artery (SMA) was also dissected but not occluded, and the hepatic artery and cystic artery were enhanced through the collateral from the SMA. Moreover, the right common iliac artery was non-enhanced (Fig. 1C). Because any symptoms of visceral ischemia such as pain, bloody stool, and/or serum biochemical abnormalities were not encountered, we diagnosed right lower limb ischemia associated with acute aortic dissection, and emergent femorofemoral bypass grafting was performed. On the following day, she complained of abdominal pain; wherein, the aspartate aminotransferase and alanine aminotransferase levels rapidly increased to $5176 \mathrm{IU} / \mathrm{L}$ and $3726 \mathrm{IU} / \mathrm{L}$, respectively. Contrasted CT demonstrated the thickened wall of the gallbladder and the pericholecystic fluid (Fig. 2), which were not found the previous day (Fig. 1A). We diagnosed visceral malperfusion with suspicious acute gallbladder necrosis, and emergent exploratory laparotomy was proposed. During laparotomy, a perforated necrotic gallbladder with a small amount of bilious ascites was found; hence, the cholecystectomy was first performed. After sufficient intraperitoneal irrigation, the inferior vena cava was exposed by mobilization and medial rotation of the right colon and duodenum. Bypass grafting from the right common iliac artery to the hepatic artery was 

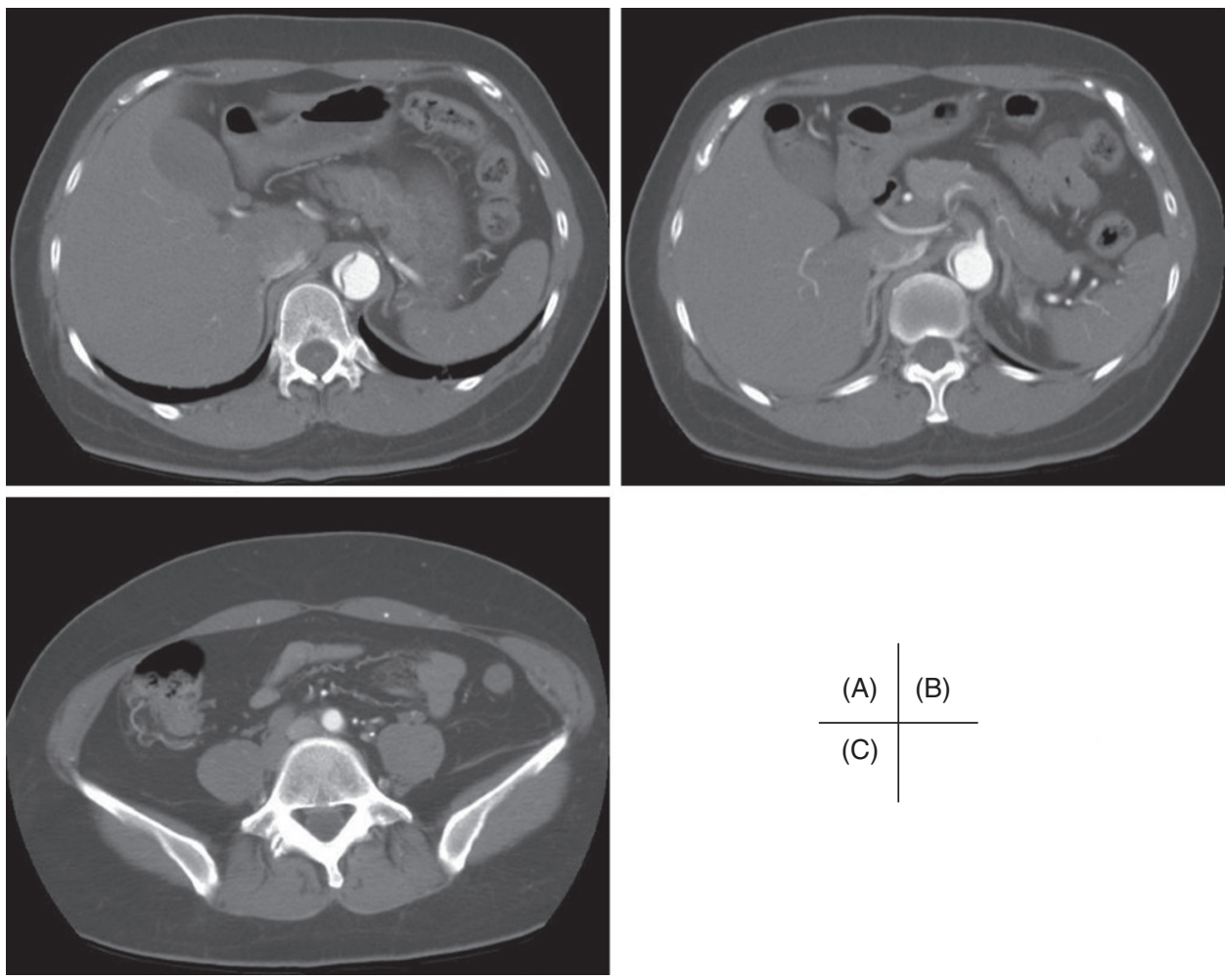

Fig. 1 Contrasted computed tomography on admission showed occlusion of the celiac artery (B) and the non-enhanced right iliac artery (C). There were no abnormal findings on the gallbladder (A).

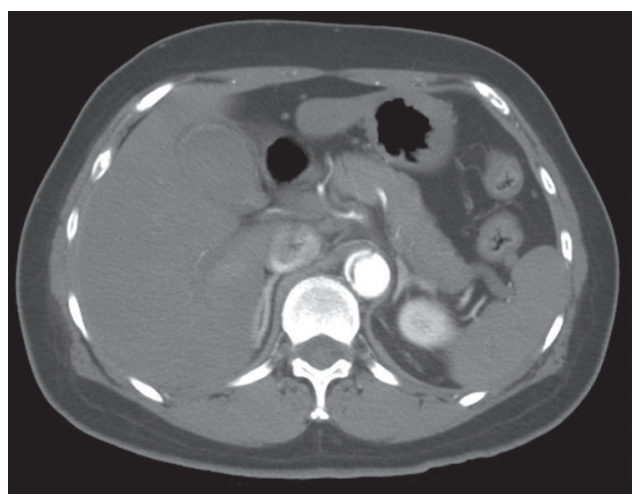

Fig. 2 Contrasted computed tomography on the day after the onset of acute aortic dissection showed the thickened wall of the gallbladder and the pericholecystic fluid.

performed using autologous saphenous vein. The graft was then positioned along the inferior vena cava. Postoperative histological examination showed acellular transmural severe necrosis of the gallbladder (Fig. 3). During the postoperative period, the aspartate aminotransferase and alanine aminotransferase levels subsided gradually to normal levels, and the abdominal pain was resolved.

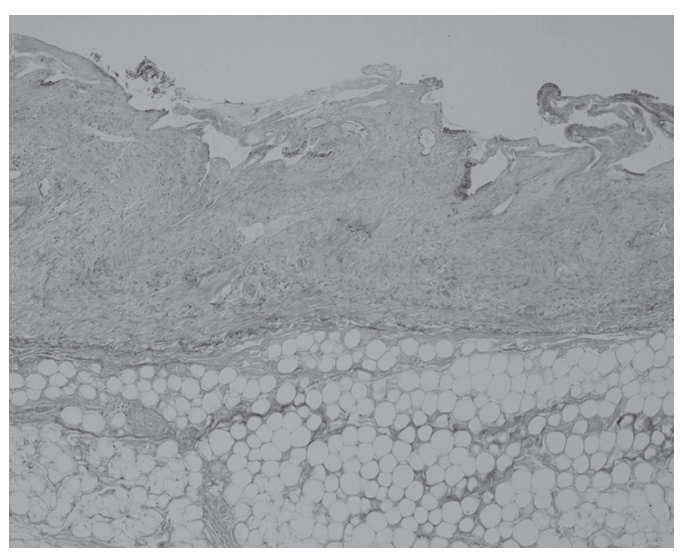

Fig. 3 Histological examination showed acellular transmural severe necrosis of the gallbladder.

Her postoperative course was uneventful, and she was discharged after 20 postoperative days.

\section{Discussion}

Although terminal organ ischemia caused by aortic branch vessel malperfusion, which occurs in about $30 \%$ 
of the patients with acute aortic dissection, ${ }^{1)}$ is not uncommon, acute gallbladder necrosis induced by acute aortic dissection is very rare. There were two papers in the review of literature that presented the case reports of acute gallbladder necrosis occurring with an acute aortic dissection. ${ }^{2,3)}$ Although it was unclear whether the visceral arteries were intact or not, histological examination that showed deep acellular necrosis of the gallbladder in both reports indicated that gallbladder ischemia played an important role. Basically, it is rare for symptoms of visceral ischemia to develop with occlusion of the CA because there are several important collateral pathways between the CA and SMA. ${ }^{4)}$ However, in our case, in addition to occlusion of the CA, the curtain-like obstruction of the orifice of the SMA by the intimal flap and conservative hypotensive therapy could lead to gallbladder ischemia. Moreover, the fact that ischemic symptom appeared in relation to the gallbladder first is interesting. It implies that the gallbladder is vulnerable to ischemia compared to other visceral organs nourished by the CA. Mehta, et al., reported that one of 31 patients, who had demonstrable gastroduodenal collateral circulation between the SMA and CA, suffered from acute acalculus cholecystitis for celiac artery coverage during endovascular thoracic aortic aneurysm repair.5) This paper also strengthens our impression about gallbladder ischemia.

Once gallbladder necrosis associated with visceral malperfusion in patients with acute aortic dissection is diagnosed, we emphasize the significance of prompt cholecystectomy because gallbladder ischemia can progress rapidly to gangrene and perforation. However, the preoperative diagnosis of gallbladder necrosis is difficult. Although CT and ultrasonography usually confirm the diagnosis with the use of the following criteria: (1) gallbladder wall thickness $\geq 4 \mathrm{~mm}$, (2) pericholecystic fluid or subserosal edema without ascites, (3) intramural gas, or (4) sloughed mucosal membrane, ${ }^{6}$ organ ischemia complicated with acute aortic dissection deteriorates rapidly and more often than not, we sometimes underestimate the severity of organ ischemia by preoperative images. Terminal visceral organ ischemia is a life-threatening complication associated with acute aortic dissection. Exploratory laparotomy for endoperitoneal observation should not be delayed in case any signs suspicious for visceral malperfusion associated with acute aortic dissection were encountered. In our case report, prompt open cholecystectomy as concomitant operation with revascularization of the celiac artery could be performed because immediate laparotomy was decided as soon as visceral malperfusion was suspected by abnormal elevation of hepatic enzymes and CT findings of thickened wall of the gallbladder and the pericholecystic fluid.

\section{Disclosure Statement}

We have no conflict of interest to disclose with respect to this material.

\section{REFERENCES}

1) Cambria RP, Brewster DC, Gertler J, et al. Vascular complications associated with spontaneous aortic dissection. J Vasc Surg 1988; 7: 199-209.

2) Roth T, Mainguene C, Boiselle JC. Acute acalculous cholecystitis associated with aortic dissection: report of a case. Surg Today 2003; 33: 633-5.

3) Sögüutlü G, Işik B, Yilmaz M, et al. Acute acalculous cholecystitis induced by aortic dissection: report of a case. Ulus Travma Acil Cerrahi Derg 2010; 16: 283-5.

4) Kornblith PL, Boley SJ, Whitehouse BS. Anatomy of the splanchnic circulation. Surg Clin North Am 1992; 72: 1-30.

5) Vaddineni SK, Taylor SM, Patterson MA, et al. Outcome after celiac artery coverage during endovascular thoracic aortic aneurysm repair: preliminary results. J Vasc Surg 2007; 45: 467-71.

6) Mirvis SE, Vainright JR, Nelson AW, et al. The diagnosis of acute acalculous cholecystitis: a comparison of sonography, scintigraphy, and CT. AJR Am J Roentgenol 1986; 147: 1171-5. 